\title{
Is there an oncological benefit to extended lymphadenectomy for muscle-invasive bladder cancer?
}

\author{
Timothy N. Clinton^, Chun Huang, Alvin C. Goh \\ Urology Service, Memorial Sloan Kettering Cancer Center, New York, NY, USA \\ Contributions: (I) Conception and design: TN Clinton, AC Goh; (II) Administrative support: None; (III) Provision of study materials or patients: TN \\ Clinton, AC Goh; (IV) Collection and assembly of data: All authors; (V) Data analysis and interpretation: All authors; (VI) Manuscript writing: All \\ authors; (VII) Final approval of manuscript: All authors. \\ Correspondence to: Alvin C. Goh, MD. Memorial Sloan Kettering Cancer Center, 1275 York Avenue, New York, NY 10065, USA. \\ Email: goha@mskcc.org.
}

\begin{abstract}
The full optimal extent of a pelvic lymph node dissection (PLND) at time of radical cystectomy (RC) has not yet been determined. The diagnostic role of LND is clear and is extremely important for identifying those who may benefit from adjuvant therapy. While retrospective analyses have demonstrated improved survival when the number of lymph nodes is increased and extended LNDs (eLNDs) are performed, these results have yet to be borne out in prospective randomized phase III trials. The recently published LEA AUO AB 25/02 trial is a promising attempt to determine the efficacy of eLND, but unfortunately falls short because of its limited design and therefore, did not demonstrate an improvement in recurrence-free survival (RFS). In an era of increased utilization of neoadjuvant chemotherapy (NAC) providing survival benefit, the ability to demonstrate improved survival with eLND is even more challenging. Currently, we are awaiting the results of SWOG S1011, expectations of achieving a positive trial with improved RFS remains unlikely.
\end{abstract}

Keywords: Lymph node excision; urinary bladder neoplasms

Submitted Jan 10, 2020. Accepted for publication May 25, 2020.

doi: 10.21037/tau-20-406

View this article at: http://dx.doi.org/10.21037/tau-20-406

Radical cystectomy (RC) with pelvic lymph node dissection (PLND) remains the standard of care for those with muscle-invasive bladder cancer. PLND excises all cancer in the bladder, pelvic organs (prostate, uterus, ovaries), and regional lymph nodes, and additionally is conducted with curative intent for patients with localized bladder cancer. Unfortunately, a large proportion of patients, even those with localized disease and treated with PLND at time of RC, will still eventually die of bladder cancer. Attempts to improve survival of localized bladder cancer has focused mainly on a complete PLND and neoadjuvant/adjuvant chemotherapy. While level 1 evidence has demonstrated improvement in survival with neoadjuvant chemotherapy (NAC) (1), surgical trials have yet to demonstrate the same level of evidence for extended LND (eLND) in localized muscle-invasive bladder cancer.

\section{Anatomic limits}

Lymphatic mapping of nodal spread for bladder cancer has demonstrated that the primary drainage sites include the internal and external iliac, obturator, and sacral lymph nodes. A multimodality study to accurately map the lymphatic drainage of the bladder used radiolabeled tracer

\footnotetext{
^ ORCID: 0000-0002-0616-6840.
} 
injections within six non-tumor bearing regions of the bladder (2). This study found that the lymphatic drainage of the bladder is complex with a median of over 24 primary landing zones including $19 \%$ that are proximal to the bifurcation of the common iliac vessels. Therefore, a limited PLND results in only a $50 \%$ resection of lymph nodes, whereas an eLND up to the inferior mesenteric artery (IMA) results in complete removal. Despite knowledge of these primary drainage sites, a study by Tarin et al. showed that $7 \%$ of patients had isolated nodal metastases above the common iliac bifurcation, the so called "skip" metastases (3). Similarly, a large institutional mapping study found that out of nearly 600 lymph node metastases, $17 \%$ were $\mathrm{pN} 3$ and $6 \%$ of positive lymph nodes were skip metastases (4). Other studies have shown that current clinical staging abilities are inadequate, as $20-27 \%$ of clinically node-negative patients who received an RC have nodal metastasis at time of surgery with upstaging (5-7). Attempts to improve clinical staging imaging capabilities with the use of PET imaging have also been of limited utility (8). Routine use of ${ }^{18} \mathrm{~F}$-FDG PET/ CT for clinically node negative patients has a sensitivity of only $7-23 \%$, thus rarely detecting metastases. The use was found to be much better for characterizing enlarged nodes to rule out lymph node metastases. Therefore, the diagnostic utility of eLND provides a vital role for staging bladder cancer, which subsequently can guide the use of adjuvant chemotherapy. At our institution, we routinely perform eLNDs (Figure 1). The eLNDs we perform encompass the aortic bifurcation (up to the level of the IMA, at the surgeon's discretion) superiorly, the genitofemoral nerve laterally, the node of Cloquet distally, and the hypogastric vessels and presacral lymph nodes posteriorly.

\section{Surrogates}

In addition to staging, lymph node status is also prognostic of cancer-specific survival (CSS) for patients with bladder cancer (9). Studies have shown that an increased number of lymph nodes removed results in improved survival and limits local recurrence $(10,11)$. While multiple studies have attempted to establish a minimum number of nodes as a surrogate for an adequate lymphadenectomy, the nodal count is widely variable and largely susceptible to processing and pathologic assessment $(12,13)$. This variability was expertly demonstrated by the studies showing that the presentation of individual packaged lymph nodes by anatomical location resulted in increased lymph node yields $(14,15)$. Subsequent studies have shown anatomic limits of dissection to be more important than lymph node counts as a surrogate for adequate resection (13). Given the unreliability and variable nature of lymph node counts, focus has shifted to determining the extent of PLND and its therapeutic role.

Currently, the American Urological Association guidelines strongly recommend performing a bilateral pelvic lymphadenectomy that includes at least the external and internal iliac and obturator lymph nodes (16). They further recommend a minimum of 12 lymph nodes for evaluation as a surrogate for the quality and completeness of the surgical dissection. The European Association of Urology guidelines acknowledge that while there is strong evidence to support performing an LND at time of RC, there is no conclusive evidence as to the optimal extent (17). The European Association of Urology guidelines do state there are data to show that eLND may improve survival after RC compared to standard/limited lymphadenectomy (level of evidence 3).

\section{Intraoperative technology for lymph node dissection}

With the limitations of clinical nodal staging and difficulty in identifying surrogates for lymph node dissection, intraoperative techniques for improving surgical LND have been developed. These techniques have been focused on sentinel node detection with cystoscopic peritumoral injections. Initial studies utilized preoperative lymphoscintigraphy and intraoperative dynamic lymphoscintigraphy using technetium (Tc-99m) (18). Unfortunately, there is nearly a $20 \%$ false-negative rate with this technique, and it is limited to micrometastatic disease due to lymphatic obstruction from bulky metastasis. With growing experience with robotic cystectomy, the use of near infrared fluorescence imaging with indocyanine green (ICG) has been explored as this technology is integrated into the DaVinci robotic system (Intuitive Surgical, Sunnyvale, CA, USA). With the injection of the ICG dye peritumorally, the initial results demonstrated safety and feasibility $(19,20)$, but larger registration studies have demonstrated less clinically useful results with $11 \%$ false negative rates and positive predictive value of $37 \%$ (21). Given the multifocality and complex lymphatic drainage from the bladder, a reliable technique to identify potential sentinel lymph node landing sites has not been established. Further, the oncologic benefit of these approaches will need to be prospectively validated to justify their routine usage and costs. 


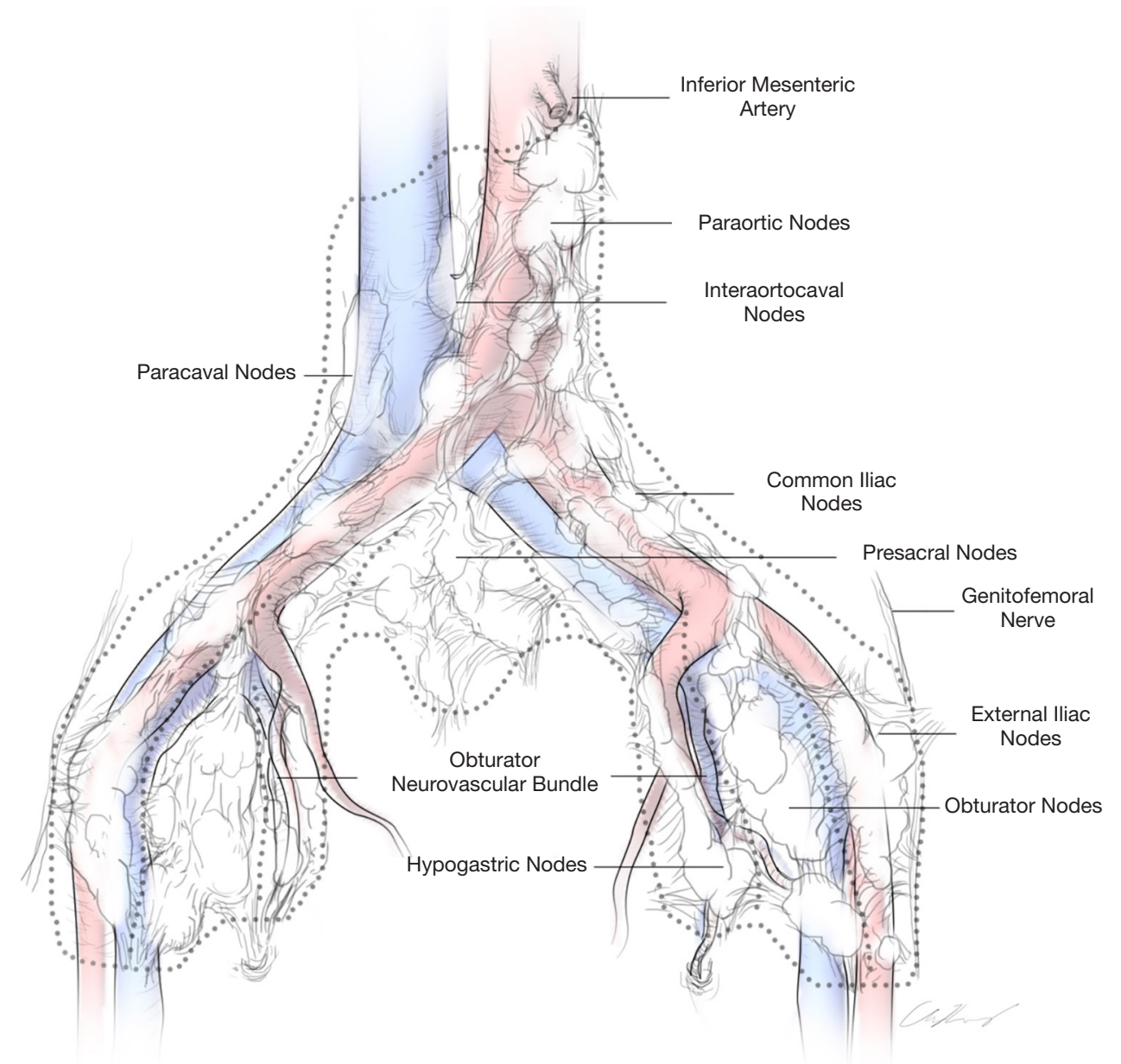

Figure 1 Extent of eLND performed at Memorial Sloan Kettering Cancer Center. eLND, extended lymph node dissection.

\section{Retrospective studies}

The evidence in support of eLND comes exclusively from retrospective analyses (Table 1). Dhar et al. retrospectively compared outcomes from two institutions with 336 patients undergoing limited LND (proximal to common iliac bifurcation) to 322 patients undergoing eLND (proximally to common iliac arteries and crossing of the ureter) (23). Analysis demonstrated a lower rate of node-positive disease and poorer outcomes for patients with node-positive and node negative disease in those with limited LND. The 5 -year recurrence-free survival (RFS) for node-positive patients was $7 \%$ in the limited LND group versus $35 \%$ in the eLND group. Tarin et al. evaluated nearly 600 patients with lymph node mapping from eLND and found that $13 \%$ of those with $\geq \mathrm{pT} 2$ disease had $\mathrm{pN} 3$ disease (3). While those with $\mathrm{pN} 3$ disease had a $25 \% 5$-year RFS, this was surprisingly not significantly different for patients with $\mathrm{pN} 1$ or $\mathrm{pN} 2$ disease. Given that $13 \%$ of $\geq \mathrm{pT} 2$ patients had $\mathrm{pN} 3$ disease, they calculated that with an observed $42 \%$ CSS at 3 years, this would result in an approximately $5 \%$ improvement in CSS through routine inclusion of common iliac lymph nodes in PLND. This 5\% improvement in CSS is very informative when evaluating the prospective clinical trials. Mandel et al. in a meta-analysis, summarized the prior studies and found that 5 -year RFS was $55 \%$ vs. $62 \%$ $(\mathrm{P}<0.001)$ in the standard vs. eLND (29). This difference of $7 \%$ was similar across all retrospective studies, helps to quantify an expected RFS benefit for eLND, and should be considered when designing prospective studies. 


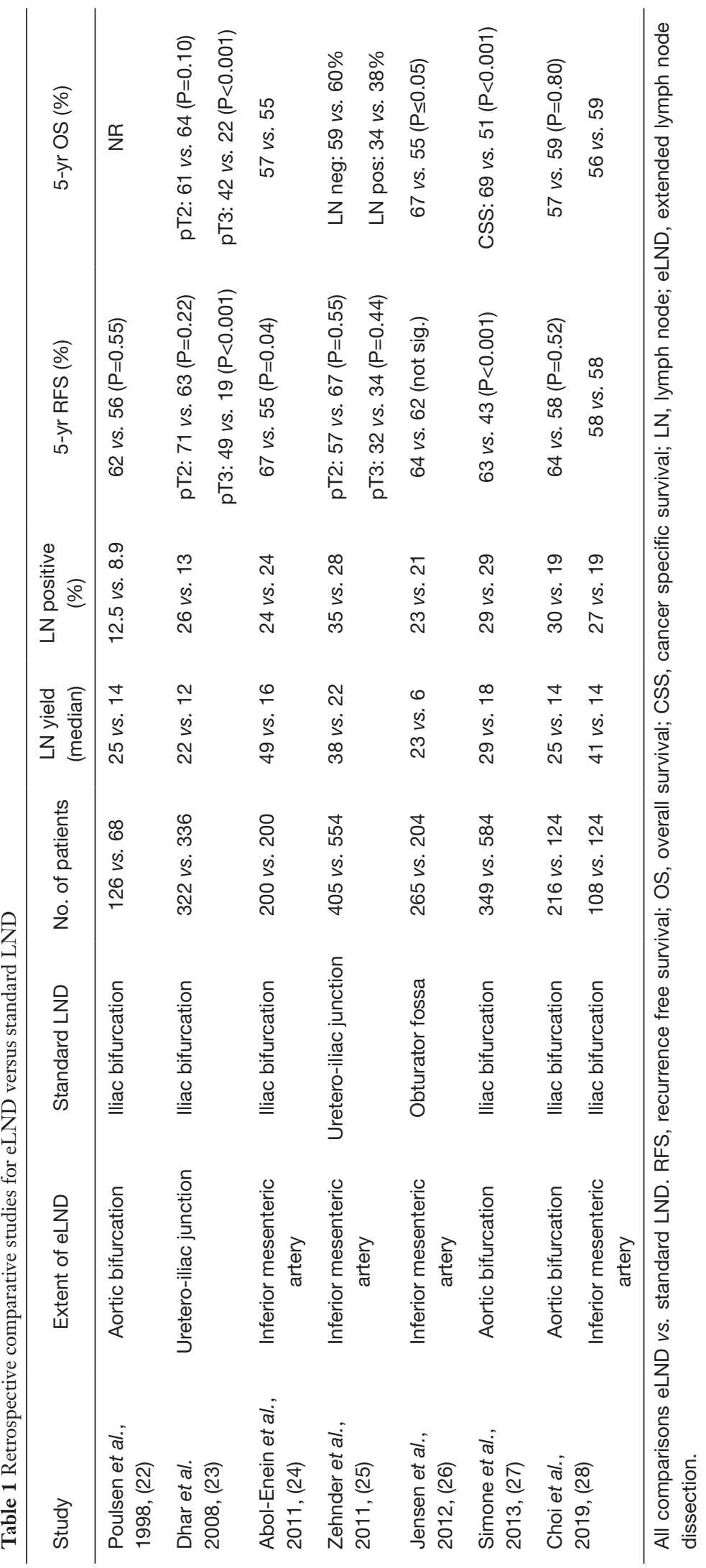




\section{Prospective clinical trials}

\section{LEA trial}

In an effort to truly determine the oncological benefit of eLND, the first prospective, randomized multicenter phase III surgical trial (LEA AUO AB 25/02) to compare limited versus eLND for improvement in RFS was recently reported by the Association for Urologic Oncology of the German Cancer Society (30). It is important to clarify terminology here. Although titled limited LND, the anatomic limits were consistent with the standard LND that the American Urology Association guidelines have defined proximally by the bifurcation of internal and external iliac artery, distally by the pelvic floor, laterally by the genitofemoral nerve, and dorsally by the obturator nerve. Uniquely, they excluded the deep obturator nodes, which are often included in standard dissections. Regardless, the eLND included the deep obturator, presacral, common iliac, paracaval/interaortocaval/paraaortic nodes up to the IMA. Given prior mapping studies, an eLND to the IMA would include all primary landing zones including skip metastases (3). The trial comprised relatively high-volume centers with experienced surgeons and included all patients with pathologic T1G3 and cT2-T4a disease undergoing RC. Non-muscle invasive tumors (T1) comprised $14 \%$ of this cohort. Given that $5-10 \%$ of pT1 patients will have nodal metastasis at RC $(3-5,13)$, the incidence of lymph node positivity would be lower and effect of the LND likely reduced. In this trial, patients were excluded from receiving NAC, which limits a potential confounder, but is not the current standard of care. While adjuvant chemotherapy was acceptable and evenly split between the two cohorts, only $28 \%$ of patients with locally advanced or node-positive disease received adjuvant treatment.

The primary endpoint of the LEA trial was RFS with secondary endpoints of CSS, overall survival (OS), complication rates, influence of adjuvant chemotherapy, and localization of tumor recurrence. The planned sample size of 400 randomized patients was based on a 5 -year RFS of $65 \%$ for the eLND and $50 \%$ for the limited lymphadenectomy, thereby expecting a $15 \%$ RFS improvement ( $\mathrm{P}<0.05$, two-sided, $80 \%$ power). Ultimately, 401 patients were randomized to limited versus eLND. In this study, eLND did show a slightly improved in 5-year RFS rate, however, this failed to meet statistical significance (65\% vs. 59\%, $\mathrm{P}=0.34)$. Secondary endpoints of CSS (76\% vs. $65 \%, \mathrm{P}=0.10)$ and $\mathrm{OS}(59 \%$ vs. $50 \%, \mathrm{P}=0.12)$ also were unable to meet statistical significance. It should be noted that although not statistically significant, eLND consistently showed a reduced risk in RFS, CSS and OS. Despite this trial not meeting its per protocol or intent-to-treat endpoints, additional insights were still gained. Analysis of the mortality and major complications between the two groups did not differ. An extended dissection was associated with a higher number of number of lymphoceles requiring intervention $(\mathrm{P}=0.04)$, albeit the numbers were low with only a total of 17 (8.6\%) patients in the eLND and 7 (3.4\%) patients in the limited lymphadenectomy groups requiring drainage within 90 days. This study also demonstrated that based on templates of eLND, a limited lymphadenectomy would have missed 21 (11\%) nodal metastases including 4 (2\%) skip metastases.

The LEA trial was not without its limitations. The trial was designed to achieve a $15 \%$ improvement in RFS, which was a lofty goal, but likely driven by an attempt at a reasonable accrual goal. Also, the inclusion of cT1G3 patients likely hindered the results as the incidence of nodal metastases or recurrence for these patients is significantly lower than the higher staged patients. There was a disproportion of $\mathrm{T} 1$ disease in the limited $v s$. eLND groups with $12 \%$ vs. $16 \%$, respectively. This also contributed to a higher rate of pathologic nodal disease in the limited lymph node dissection as compared to the eLND (28\% vs. $22 \%$ ), which is likely skewed due to the pT1 patients in the eLND cohort. While the primary and secondary endpoints were not achieved, the trend towards reduced risk in RFS, CSS, and OS with no difference in mortality or high-grade complications provides some support for the inclusion of an eLND with RC.

\section{SWOG trial}

In the United States, a second randomized prospective phase III trial has also completed accrual but is awaiting follow-up for final analysis and publication (SWOG S1011; ClinicalTrials.gov number, NCT01224665). Like the LEA trial, the SWOG trial studied only high-volume centers and surgeons but mandated a credentialing process with intraoperative photos (31). This trial had inclusion criteria that differed from the LEA trial by allowing patients to undergo NAC (56\% of patients received), as well as limiting enrollment to patients with cT2-4a disease, and excluding patients with $\mathrm{pT} 1$. While the inclusion of NAC is consistent with routine standard of care, this will likely confound and reduce the benefit of surgical resection and increase the number needed to treat. The study has a more reasonable 
expected $10 \%$ improvement from $55 \%$ to $65 \%$ in 3 -year RFS for eLND at $85 \%$ power with 620 randomized patients given a $10 \%$ ineligible drop-out expected rate. Patients were randomized based on receipt of NAC, clinical stage and performance status. Currently, the trial is ongoing, and we eagerly await the results.

There may be some challenges in the SWOG trial reaching its primary endpoint of a $10 \%$ improvement in 3-year RFS. As previous meta-analyses of retrospective studies have suggested (29), a dissection of lymph nodes above the bifurcation of the common iliac would likely result in an improvement in RFS of 5-7\%. This result is similar to the survival benefit shown for NAC demonstrated in several randomized trials $(1,32)$. Unfortunately, to demonstrate a 3-year CSS improvement of $5 \%$ with a power of $80 \%$ using a one-sided stratified logrank test with alpha $=0.025$, would require accrual of more than 2,000 patients (4). This CSS improvement from Tarin et al. included 23\% who received NAC, but the difference in survival did not change even when excluding those patients. Unlike the German trial, the SWOG study will be limited by the inclusion of NAC prior to RC and LND. With increasing use of NAC, the treatment effect of LND could be reduced and the cohort size may not be sufficient to demonstrate significant differences. Although there was no specified expected percentage of patients receiving NAC, interim analyses of the SWOG study reported some of the highest usage rates seen in prior studies (33). Additionally, randomization of patients is done intra-operatively such that any clinically suspicious nodes at or above the bifurcation of the common iliac vessels are allowed to be resected for frozen section prior to randomization as failure to excise lymph nodes with known cancer involvement would be a substantial deviation from standard of care. Removal of suspicious nodes could also reduce the impact of the thorough node dissection.

In a subset analysis of SWOG 8710, an adequate lymph node dissection with $\geq 10$ nodes was associated with an OS benefit regardless of receipt of NAC (10). From the National Cancer Database [2004-2012], a recent study looked at number of lymph nodes during lymphadenectomy for RC in those who received NAC compared to those who did not (34). Interestingly, they found that for those who did not receive NAC, adequate lymph node dissection of $\geq 10$ nodes was associated with an OS benefit for all stages of disease, but there was no improvement in OS for those who actually received NAC. Given that over half of the patients enrolled in the SWOG S1011 trial belong to the latter group, demonstrating even a $5 \%$ improvement in RFS in those who have undergone NAC may be unlikely.

\section{Future directions}

Designing and implementing surgical trials is extremely difficult and onerous, which makes randomized phase III trials like the LEA trial and SWOG S1011 impressive. Surgical trials are inherently biased towards surgeon technique and center differences; SWOG S1011 took a novel approach to control for this heterogeneity. The trial vetted high-volume surgeons through an enrollment period which conducted intraoperative photography to ensure standardization for extent and completeness of surgical dissection. While this level of standardization is admirable, development of future surgical trials to address the question of extent of pelvic lymphadenectomy are unlikely given the logistics involving time and cost of accruing enough patients. The current trials have been powered for RFS. While survival may be more clinically relevant, the sample size and time for follow-up required present significant feasibility challenges. Given the minimal risk and time to perform an eLND, one may consider whether the question remains relevant. With a recent increase of robotic RC (35), performing an eLND robotically has been shown to be technically feasible without increasing complications $(36,37)$.

With advances in immunotherapy agents, the focus to improve recurrence and survival outcomes has shifted to novel neoadjuvant systemic therapies. As seen in SWOG S1011, there has been an increase in the use of NAC, but a recent phase II trial (PURE-01) has reported the use of neoadjuvant immunotherapy with pembrolizumab (38). The early results are promising as they demonstrate a $42 \%$ complete response rate and pathologic downstaging in $54 \%$ of patients. Two patients in PURE-01 were considered clinical N1 based on preoperative PET imaging, but the enrollment of these patients in neoadjuvant trials remains controversial with such locally advanced disease. With the high rates of downstaging, there may be a role for including clinically node positive patients for future consideration in immunotherapy trials prior to surgical resection. For those with pathologic positive nodal disease, the use of adjuvant immunotherapy is being investigated with multiple phase III randomized trials including pembrolizumab (AMBASSADOR; ClinicalTrials. gov number, NCT03244384), nivolumab (CheckMate 274; ClinicalTrials.gov number, NCT02632409) and atezolizumab (IMvigor010; ClinicalTrials.gov number, NCT02450331). Recent press release from Roche, has tempered excitement 
for adjuvant atezolizumab with the primary endpoint of disease-free survival for IMvigor010 was not reached and is awaiting formal publication results (39).

Given these findings, current trials are now logically combining chemotherapy and immunotherapy with gemcitabine, cisplatin, and atezolizumab (ClinicalTrials.gov number, NCT02989584) in hopes of obtaining even more impressive response rates. With the success of neoadjuvant systemic therapies and improvements in next generation sequencing of tumors, our ability to distinguish the tumors that will respond to chemotherapy has led to consideration of bladder preservation. This has led to recent trials to evaluate in the risk stratification for radical surgery in the hopes of allowing those with deleterious DNA damage response gene alterations to undergo chemotherapy without cystectomy (ClinicalTrials.gov number, NCT03609216).

\section{Conclusions}

Given the current state of evidence with largely retrospective analyses and a phase III trial, with limitations in its design, the oncologic efficacy of an eLND needs further investigation. From meta-analyses, we know that a greater number of lymph nodes removed and eLND are associated with improvements in RFS and CSS. However, most of these studies did not include patients who now routinely undergo NAC. While not statistically significant, there was a trend that suggests that survival in the LEA may have been improved with eLND and thus eLND warrants further evaluation. Importantly, the trial showed no significant difference in mortality or morbidity by performing an eLND compared to standard LND. We await the results of the SWOG S1011 trial with the hope of further clarifying the role of eLND. However, the findings may be limited by sample size and the increasing usage of upfront systemic therapy. An eLND can be done safely, provides important staging and prognostic information, and appears to provide therapeutic benefit. Thorough and complete resection of the bladder as well as surrounding lymphatic tissue remain a central tenet in the optimal surgical management of invasive bladder cancer.

\section{Acknowledgments}

Funding: Supported by the Sidney Kimmel Center for Prostate and Urologic Cancers and the National Institutes of Health/National Cancer Institute to Memorial Sloan Kettering Cancer Center through the Cancer Center
Support Grant, award number P30 CA008748, NIH/ HCATS Grant UL1-TR002384 and by SPORE in Bladder Cancer P50 CA221745.

\section{Footnote}

Provenance and Peer Review: This article was commissioned by the Guest Editors (Ja Hyeon Ku, Ho Kyung Seo, Seok Ho Kang) for the series "Muscle-Invasive Bladder Cancer" published in Translational Andrology and Urology. The article has undergone external peer review.

Peer Review File: Available at http://dx. doi. org/10.21037/ tau-20-406

Conflicts of Interest: All authors have completed the ICMJE uniform disclosure form (available at http://dx. doi. org/10. 21037/tau-20-406). The series "Muscle-Invasive Bladder Cancer" was commissioned by the editorial office without any funding or sponsorship. Dr. ACG reports to receive consulting fee from Medtronic, outside the submitted work. The authors have no other conflicts of interest to declare.

Ethical Statement: The authors are accountable for all aspects of the work in ensuring that questions related to the accuracy or integrity of any part of the work are appropriately investigated and resolved.

Open Access Statement: This is an Open Access article distributed in accordance with the Creative Commons Attribution-NonCommercial-NoDerivs 4.0 International License (CC BY-NC-ND 4.0), which permits the noncommercial replication and distribution of the article with the strict proviso that no changes or edits are made and the original work is properly cited (including links to both the formal publication through the relevant DOI and the license). See: https://creativecommons.org/licenses/by-nc-nd/4.0/.

\section{References}

1. Grossman HB, Natale RB, Tangen CM, et al. Neoadjuvant chemotherapy plus cystectomy compared with cystectomy alone for locally advanced bladder cancer. N Engl J Med 2003;349:859-66.

2. Roth B, Wissmeyer MP, Zehnder P, et al. A new multimodality technique accurately maps the primary lymphatic landing sites of the bladder. Eur Urol 
2010;57:205-11.

3. Tarin TV, Power NE, Ehdaie B, et al. Lymph nodepositive bladder cancer treated with radical cystectomy and lymphadenectomy: effect of the level of node positivity. Eur Urol 2012;61:1025-30.

4. Leissner J, Ghoneim M, Abol-enein H, et al. Extended radical lymphadenectomy in patients with urothelial bladder cancer: results of a prospective multicenter study. J Urol 2004;171:139-44.

5. Vazina A, Dugi D, Shariat SF, et al. Stage specific lymph node metastasis mapping in radical cystectomy specimens. J Urol 2004;171:1830-4.

6. Millikan R, Dinney CP, Swanson D, et al. Integrated therapy for locally advanced bladder cancer: final report of a randomized trial of cystectomy plus adjuvant $\mathrm{M}-\mathrm{VAC}$ versus cystectomy with both preoperative and postoperative M-VAC. J Clin Oncol 2001;19:4005-13.

7. Shariat SF, Palapattu GS, Karakiewicz PI, et al. Discrepancy between clinical and pathologic stage: impact on prognosis after radical cystectomy. Eur Urol 2007;51:137-49; discussion 149-51.

8. Dason S, Wong NC, Donahue TF, et al. Utility of Routine Preoperative 18 F-Fluorodeoxyglucose Positron Emission Tomography-Computerized Tomography in Identifying Pathological Lymph Node Metastases at Radical Cystectomy. J Urol 2020;204:254-9.

9. International Bladder Cancer Nomogram Consortium; Bochner BH, Kattan MW, et al. Postoperative nomogram predicting risk of recurrence after radical cystectomy. J Clin Oncol 2006;24:3967-72.

10. Herr HW, Faulkner JR, Grossman HB, et al. Surgical factors influence bladder cancer outcomes: a cooperative group report. J Clin Oncol 2004;22:2781-9.

11. Wright JL, Lin DW, Porter MP. The association between extent of lymphadenectomy and survival among patients with lymph node metastases undergoing radical cystectomy. Cancer 2008;112:2401-8.

12. Koppie TM, Vickers AJ, Vora K, et al. Standardization of pelvic lymphadenectomy performed at radical cystectomy. Cancer 2006;107:2368-74.

13. Dorin RP, Daneshmand S, Eisenberg MS, et al. Lymph node dissection technique is more important than lymph node count in identifying nodal metastases in radical cystectomy patients: a comparative mapping study. Eur Urol 2011;60:946-52.

14. Bochner BH, Cho D, Herr H, et al. Prospectively packaged lymph node dissections with radical cystectomy: evaluation of node count variability and node mapping. J
Urol 2004;172:1286-90.

15. Zehnder P, Moltzahn F, Mitra AP, et al. Radical cystectomy with super-extended lymphadenectomy: impact of separate vs en bloc lymph node submission on analysis and outcomes. BJU Int 2016;117:253-59.

16. Chang SS, Bochner BH, Chou R, et al. Treatment of nonmetastatic muscle-invasive bladder cancer: AUA/ASCO/ ASTRO/SUO guideline. J Urol 2017;198:552-9.

17. Witjes JA, Lebret T, Comperat E, et al. Updated 2016 EAU Guidelines on muscle-invasive and metastatic bladder cancer. Eur Urol 2017;71:462-75.

18. Liedberg F, Chebil G, Davidsson T, et al. Intraoperative sentinel node detection improves nodal staging in invasive bladder cancer. J Urol 2006;175:84-8.

19. Schaafasm BE, Verbeek FPR, Elzevier HW, et al. Optimization of sentinel lymph node mapping in bladder cancer using near-infrared fluorescence imaging. J Surg Oncol 2014;110:845-50.

20. Manny TB, Hemal AK. Fluorescence-enhanced robotic radical cystectomy using unconjugated indocyanine green for pelvic lymphangiography, tumor marking, and mesenteric angiography: the initial clinical experience. Urology 2014;83:824-30.

21. Polom W, Markuszewski M, Cytawa W, et al. Fluorescent versus radioguided lymph node mapping in bladder cancer. Clin Genitourin Cancer 2017;15:e405-9.

22. Poulsen AL, Horn T, Steven K. Radical cystectomy: extending the limits of pelvic lymph node dissection improves survival for patients with bladder cancer confined to the bladder wall. J Urol 1998;160:2015-9; discussion 2020.

23. Dhar NB, Klein EA, Reuther AM, et al. Outcome after radical cystectomy with limited or extended pelvic lymph node dissection. J Urol 2008;179:873-8.

24. Abol-Enein H, Tilki D, Mosbah A, et al. Does the extent of lymphadenectomy in radical cystectomy for bladder cancer influence disease-free survival? A prospective single-center study. Eur Urol 2011;60:572-7.

25. Zehnder P, Studer UE, Skinner EC, et al. Super extended versus extended pelvic lymph node dissection in patients undergoing radical cystectomy for bladder cancer: a comparative study. J Urol 2011;186:1261-8.

26. Jensen JB, Ulhøi BP, Jensen KM. Extended versus limited lymph node dissection in radical cystectomy: Impact on recurrence pattern and survival. Int J Urol 2012;19:39-47.

27. Simone G, Papalia R, Ferriero M, et al. Stage-specific impact of extended versus standard pelvic lymph node dissection in radical cystectomy. Int J Urol 2013;20:390-7. 
28. Choi SY, You D, Hong B, et al. Impact of lymph node dissection in radical cystectomy for bladder cancer: how many vs how far? Sur Oncol 2019;30:109-16.

29. Mandel P, Tilki D, Eslick GD. Extent of lymph node dissection and recurrence-free survival after radical cystectomy: a meta-analysis. Urol Oncol 2014;32:1184-90.

30. Gschwend JE, Heck MM, Lehmann J, et al. Extended Versus Limited Lymph Node Dissection in Bladder Cancer Patients Undergoing Radical Cystectomy: Survival Results From a Prospective, Randomized Trial. Eur Urol 2019;75:604-11.

31. Lerner SP, Svatek RS. What is the standard of care for pelvic lymphadenectomy performed at the time of radical cystectomy. Eur Urol 2019;75:612-4.

32. Neoadjuvant cisplatin, methotrexate, and vinblastine chemotherapy for muscle-invasive bladder cancer: a randomised controlled trial. International Collaboration of Trialists. Lancet 1999;354:533-40.

33. Lerner S, Tangen CM, Svatek RS, et al. MP65-02 a phase III surgical trial to evaluate the benefit of a standard versus an extended pelvic lymphadenectomy performed at time of radical cystectomy for muscle invasive urothelial cancer: SWOG S10-11 (NCT\#01224665). J Urol 2015;193:e807. 34. von Landenberg N, Speed JM, Cole AP, et al. Impact of

Cite this article as: Clinton TN, Huang C, Goh AC. Is there an oncological benefit to extended lymphadenectomy for muscle-invasive bladder cancer? Transl Androl Urol 2020;9(6):2956-2964. doi: 10.21037/tau-20-406 adequate pelvic lymph node dissection on overall survival after radical cystectomy: a stratified analysis by clinical stage and receipt of neoadjuvant chemotherapy. Urol Oncol 2018;36:78.e13-78.e19.

35. Bachman AG, Parker AA, Shaw MD, et al. Minimally invasive versus open approach for cystectomy: trends in the utilization and demographic or clinical predictors using the National Cancer Database. Urology 2017;103:99-105.

36. Parekh DJ, Reis I, Castle EP, et al. Robot-assisted radical cystectomy versus open radical cystectomy in patients with bladder cancer (RAZOR): an open-label, randomised, phase 3, non-inferiority trial. Lancet 2018;391:2525-36.

37. Bochner BH, Dalbagni G, Sjoberg DD, et al. Comparing open radical cystectomy and robot-assisted laparoscopic radical cystectomy: a randomized clinical trial. Eur Urol 2015;67:1042-50.

38. Necchi A, Anichini A, Raggi D, et al. Pembrolizumab as neoadjuvant therapy before radical cystectomy in patients with muscle-invasive urothelial bladder carcinoma (PURE01): an open-label, single-arm, phase II study. J Clin Oncol 2018;36:3353-60.

39. Roche provides an update on Phase III study of Tecentriq in people with muscle-invasive urothelial cancer. Basel: Roche; 2020. 